\title{
JULES VERNE EN BOHÊME (1870-1900) : SES PREMIERS TRADUCTEURS ET LEUR STATUT SOCIO-PROFESSIONNEL'
}

\author{
Zuzana Raková \\ Université Masaryk de Brno \\ République tchèque \\ rakovaz@seznam.cz
}

doi.org/10.15452/SR.2020.20.0004

Résumé. L'article présente les auteurs des premières traductions tchèques des romans de Jules Verne, publiées au XIXe siècle, entre 1870 et 1900 . L'étude cherche à répondre à certaines questions concernant l'histoire externe de la traduction ou « l'archéologie de la traduction » dans les termes d'Anthony Pym : quels étaient les traducteurs des premiers romans de Jules Verne présentés au public tchèque, quels titres introduisirent-ils, de quel statut bénéficièrent-ils en tant que professionnels de la traduction? Cette étude fondée sur la méthode statistique laisse volontiers de côté la stratégie de traduction, sujet très vaste qui nécessiterait d'autres recherches approfondies.

Mots clés. Jules Verne. Traducteurs tchèques. Archéologie et sociologie de la traduction.

\begin{abstract}
The First Czech Translators of Jules Verne (1870-1900) in Bohemia and their Socio-professional Status. The article presents the authors of the first Czech translations of the novels of Jules Verne, published in the $19^{\text {th }}$ century, between 1870 and 1900 . The study seeks to answer several questions concerning the external history of translation or archaeology of translation in terms of Anthony Pym, for example who were the translators of the first novels of Jules Verne presented to the Czech public and which translations did they introduce? What status did the translators of this literature for the youth enjoy at the time? The study is based on the statistical method and leaves aside the translation strategy, a very large subject that would require further detailed research.
\end{abstract}

Keywords. Jules Verne. Czech translators. Archaeology and sociology of translation.

1 Cet article parait dans le cadre du projet de recherche alloué sur les fonds de la Faculté des Lettres de l'Université Masaryk de Brno pour l'année 2018 (MUNI/21/RAK/2018 Traductions tchèques du français 1890-1914 : le droit d'auteur et la traduction, la sociologie et l'économie du métier du traducteur). Nous tenons à remercier notre collègue Christophe Cusimano de l'Université Masaryk pour la correction linguistique du texte. 


\section{Introduction}

Le présent article fait suite à notre étude précédente, Les premières traductions tchèques de Jules Verne (1870-1900) : archéologie de la traduction (Raková, 2019), s’appuie sur un corpus identique et cherche à répondre à la question suivante : «Quels ont été les traducteurs qui se sont consacrés à la découverte des romans contemporains de cet auteur pour le public tchèque, et quel était leur statut socioprofessionnel ? ». Notre attention sera focalisée sur la position que représentait la traduction dans l'éventail des activités professionnelles des traducteurs concernés. Nous nous concentrerons également sur la stabilité d'une collaboration entre traducteur et éditeur.

\section{Corpus et méthodologie}

Notre recherche s'appuie sur une des bases de données de la Bibliothèque nationale de Prague, le Catalogue collectif tchèque (SKC), dans laquelle nous avons lancé une recherche en utilisant les filtres suivants : auteur « Jules Verne », langue de l'original « fre », langue du document «cze », date de la parution « 1870-1900 », support « livre ». Nous avons obtenu quatre-vingt-dix-huit résultats, dont huit doublets. Nous avons retenu pour nos statistiques les données sans doublets, en calculant chaque volume comme une unité.

\subsection{Le cadre socioéconomique et législatif de la publication du livre}

Si nous prenons comme source d'inspiration les questions fondamentales de l'histoire de la traduction (Pym, 1998 : 20), nous considérons comme important, conformément à Pym (1998: 5), de nous pencher sur les acteurs du processus de la traduction. Dans notre étude précédente (Raková, 2019), nous avons cherché des réponses sur les éditeurs et leur approche vis-à-vis des collaborateurs - traducteurs. Avant d'aborder la catégorie des traducteurs, nous résumerons les normes préliminaires (Toury, 1978: 83-100, 1995:52-69), permettant de rendre compte de certains critères et conditions externes comme la politique de la traduction ainsi que la voie d'accès aux textes originaux et la production des titres par les traducteurs. Tous les acteurs impliqués dans le processus de la production du livre traduit évoluaient dans le cadre politique, socioéconomique, législatif et culturel de l'époque. Dans la monarchie austro-hongroise du dernier tiers du XIX siècle, on ne peut mentionner aucune politique d'État relative à la publication des textes littéraires. La sélection des œuvres à traduire dépendait du choix individuel de l'éditeur et parfois, de l'initiative des traducteurs eux-mêmes, dans le cas des petites maisons d'édition notamment. ${ }^{2}$ Les programmes éditoriaux reflétaient fortement le goût des lecteurs - qu'ils contribuaient à former par la même voie -, et bien évidemment des limitations telles que la censure a posteriori, qui attribuait la responsabilité quant aux titres publiés à l'éditeur, par le biais des pertes économiques réalisées si le titre était interdit et donc retiré de la vente (Wögerbauer, 2015 : 48-49, 485).

La voie d'accès aux originaux était directe, comme l'a prouvé notre corpus des romans de Jules Verne (Raková, 2019), ce qui se révélait conforme à l'évolution progressive de l'enseignement du français durant la période en question (Raková, 2011 : 31-35), phénomène qui favorisait les traductions directes du français.

Le statut du traducteur peut être examiné par l'intermédiaire du nombre de traductions anonymes, en comptabilisant celles ne mentionnant aucune indication sur l'identité du traducteur ou une indication incomplète par le biais des initiales du nom. On peut s'attendre à une plus grande fréquence de traducteurs anonymes dans le cas du corpus de littérature pour la jeunesse, 
qui à l'époque occupait la périphérie du système littéraire (Even-Zohar, 1990: 46-48) tchèque par rapport à la littérature adressée aux lecteurs adultes.

\subsection{Les acteurs de la traduction - les traducteurs}

On peut diviser les traducteurs selon plusieurs critères : a) selon que le nom du traducteur est renseigné dans le livre (sur la page du titre) ou pas (traducteur connu vs. traducteur anonyme), éventuellement par les initiales seulement (traducteur semi-anonyme) ; b) selon l'importance de la traduction parmi les activités professionnelles : métier de la traduction exclusif ou principal (traducteur professionnel), métier de la traduction combiné avec un autre métier de l'édition ou de la presse (traducteur semi-professionnel, qui travaille de surcroît comme rédacteur, correcteur, journaliste et écrivain), métier de la traduction combiné à un métier civique principal non lié à l'espace littéraire (traducteur occasionnel, qui exerce principalement un métier civique, p. ex. celui de fonctionnaire, de professeur ou autre) ; c) selon la place du traducteur dans le canon littéraire d'aujourd'hui : traducteur anonyme dans le sens où son nom n'est aujourd'hui pas connu puisque non diffusé par l'institution littéraire, et traducteur célèbre (mentionné dans les encyclopédies littéraires de référence et donc inclus dans le canon littéraire). Quant au point c), nous nous posons la question fondamentale : y a-t-il dans le dictionnaire encyclopédique de la littérature tchèque (LČL 1-4, 1985-2008) de simples traducteurs ? Combien parmi ceux qui y figurent étaient dans le même temps écrivains, poètes, dramaturges, ou d'autres personnes actives dans la vie culturelle? Autrement dit, est-ce possible d'être intégré dans le canon littéraire uniquement en tant que traducteur?

Pour répondre à ces questions, nous examinerons les profils des traducteurs et adaptateurs dont le nom était indiqué sur les traductions ou qu'il était possible de déterminer grâce à d'autres sources. Le tableau, dressé sur la base des données du Catalogue collectiftchèque (SKC) et du Dictionnaire encyclopédique de la littérature tchèque (LČL 1-4), contient pour chaque traducteur l'indication du nombre de volumes traduit de Verne, rééditions incluses. Le système de classement des traducteurs s'effectue chronologiquement selon leurs dates de naissance; les traductions anonymes et les adaptations ${ }^{3}$ sont placées à la fin.

\section{TRADUCTEURS}

Pavel J. Šulc, 1828-1897. (LČL 4, 2008 : 808-809) S

Auteur d'ouvrages pour la jeunesse et traducteur / adaptateur du polonais, de l'allemand et du français des romans et nouvelles historiques, de proses pédagogiques pour les enfants et la jeunesse, et de quelques romans de Jules Verne ; il commença sa carrière professionnelle comme professeur dans une école secondaire (école technique de Liberec, de 1853 à 1859), puis devint journaliste, rédacteur, éditeur (par exemple chez Alois Hynek, où il dirigea plusieurs séries d'édition pour la jeunesse). Il a traduit p. ex. Robinson Crusoe de l'anglais. Il signait ses œuvres par les initiales P. J. Š. ou P. Š. T-ý. Parmi les romans de Verne publiés avant 1900, il a adapté quatre titres et a traduit un roman sous le nom P. J. Š. (voir plus loin).

Robert Nápravník (pseudonyme J. Drn), 1839-1877. (LČL 3, 2000 : 402) S

Journaliste, traducteur de textes dramatiques et de romans du français et de l'italien ; il obtint un

3 Nous avons classifié comme adaptations les volumes qui n’étaient pas explicitement désignés comme traductions dans le paratexte de l'édition tchèque. Ainsi avons-nous répertorié dans cette catégorie les volumes avec de telles indications dans le tirage comme : « dle J. Verne-a vzdělal [selon J. Verne a fait] » ou « dle J. Verne-a volně vzdělal [selon J. Verne a fait librement] », suivies du nom de l'adaptateur. Dans un cas (voir le tableau ci-dessus), le contexte déterminait clairement qu'il s'agissait d'une adaptation dramatique, destinée pour la scène. 
doctorat en droit en 1867; parmi ses traductions du français, on trouve L'Homme qui rit et Les travailleurs de la mer de Victor Hugo ou Gringoire (1873) de Théodore de Banville. Parmi les romans de Verne publiés avant 1900, il a traduit :

Autour de la Lune, 1870 / Cesta kolem Měsíce, 1870, Nákladem spolku pro vydávání laciných knih českých

Le Tour du monde en quatre-vingts jours, 1873 / Cesta kolem světa za osmdesáte dní : cestopisný román, 1873, Nákladem spolku pro vydávání laciných knih českých

Cinq semaines en ballon, 1863 / Pět neděl v ballóně : výskumná cesta tř́ Angličanů po Africe, 1874, Nákladem spolku pro vydávání laciných knih českých.

Jan Vincenc Diviš (pseudonyme Jan Čeněk), 1848-1923. O

Entre 1895 et 1908 utilisait le titre de noblesse chevalier - rytír Čistecký ze Šerlinku. Inventeur, fabricant de sucre, écrivain, auteur de travaux historiques, de mémoires, de livres sur l'industrie sucrière, traducteur, maire de Přelouč entre 1914 et 1919. Parmi les romans de Verne publiés avant 1900, il a traduit :

Aventures de trois Russes et de trois Anglais dans l'Afrique australe, 1872 / Dobrodružství tř́ Rusův a tři Angličanuv v jižní Africe, 1875, Nákladem spolku pro vydávání laciných knih českých.

Louis Schmidt-Beauchez, 1848-1912. (LČL 4, 2008 : 115-116) S

Traducteur (principalement pour les journaux) de quelques prosateurs français de la $2^{\mathrm{e}}$ moitié du $\mathrm{XIX}^{\mathrm{e}}$ s., professeur privé de français et d'allemand, médiateur des relations franco-tchèques, correspondant à Paris de Národní listy (1893-1898), puis correspondant pragois du journal parisien Le Temps ; journaliste, auteur occasionnel prosaïque et dramatique, auteur de manuel de français.

Parmi les romans de Verne publiés avant 1900, il a traduit :

Le Docteur Ox, 1874 / Doktor Ox, 1877, 1885, 1893, J. Otto, 3 éditions.

František Brábek, 1848-1926. (LČL 1, 1985 : 288) P

Traducteur assermenté de hongrois, traducteur occasionnel de français. Parmi les romans de Verne publiés avant 1900, il a traduit :

Île mystérieuse, 1874-5 / Tajemný ostrov, 3 vol. en 1878, J. Otto, 1 vol. en 1896, J. Otto.

Jan Zdeněk Veselý / J. Veselý, 1850-1890. (LČL 4, 2008 : 1300-1301) O

Parmi les romans de Verne publiés avant 1900, il a traduit :

Michel Strogoff, 1876 / Carův kurýr I, II, 2 vol., 1883, « sans éditeur 》

Un drame au Mexique, 1851, publié 1876 / Drama v Mexiku, 1884, Lauermann.

Václav Beneš Šumavský, 1850-1934. (LČL 1, 1985 : 196-197) O

Rédacteur dans différents journaux et traducteur. Parmi les romans de Verne publiés avant 1900, il a traduit :

Les Enfants du capitaine Grant, 1868 / Děti kapitána Granta, 1899, Vilímek, autorizované vyd., illustré par Josef Ulrich et Édouard Riou.

J. Al. U. = Jan Alois Unzeitig, 1853-1907. (LČL 4, 2008 : 1123-1124) S

Professeur et traducteur du français et du russe des prosateurs contemporains ; il travailla tout d'abord pour des revues et puis notamment pour les éditeurs des livres bon marché de I. L. Kober et E. Beaufort. Son fils Vítězslav Unzeitig devient un traducteur renommé et systématique des romans de Jules Verne. Parmi les romans de Verne publiés avant 1900, il a traduit :

Le Rayon vert, 1882 / Zelený paprsek, 1884, A. Lauermann.

Il a aussi traduit l'École des robinsons, remaniée plus tard par son fils Vítězslav.

Jan J. Benešovský-Veselý, 1854-1905. (LČL 1, 1985 : 203-204) O

Avant tout journaliste, mais aussi traducteur. Parmi les romans de Verne publiés avant 1900, il a traduit :

Le Chancellor, 1875 / Chancellor: deník cestujícího J. R. Kazallona, 1883, A. Lauermann. 
Jan Wagner, 1856-1905. (LČL 4, 2008: 1562-1563) S

Aussi sous les pseudonymes : Albín Straka, Antonín Koudelka.

Auteur de livres de voyages (il a enseigné l'histoire et la géographie en Bulgarie pendant cinq ans), journaliste, rédacteur (Národní politika, Matice lidu), correcteur et traducteur, surtout du russe, aussi du bulgare, de l'anglais et du français.

Parmi les romans de Verne publiés avant 1900, il a traduit :

Les Cinq Cents Millions de la Bégum, 1879 / Ocelové město, 1895, Vilímek, illustré par L. Benett

L'île à hélice, 1895 / Plující ostrov, 1896 ou 1897, Vilímek, illustré par L. Benett

Voyage au centre de la Terre, 1864 / Do středu Země, 1896 ou 1897, Vilímek, illustré par V. Černý.

Comme Albín Straka, il a traduit :

Face au drapeau, 1896 / Vynález zkázy, 1896 ou 1897, Vilímek, illustré par L. Benett.

Comme Antonín Koudelka, il a traduit :

Île mystérieuse, 1874-5 / Tajuplný ostrov, 1896 ou 1897, Vilímek, illustré par Jules Férat. Le Catalogue collectif indique Alois Koudelka comme traducteur de ce roman, mais selon le LČL 2 (1993 : 894-895), Alois Koudelka, 1861-1942, prêtre et traducteur polyglotte, n'est pas indiqué comme traducteur de Verne. Le livre contenant seulement l'indication A. Koudelka, nous attribuons cette traduction à Jan Wagner (pseudonyme Antonín Koudelka).

Václav Patejdl, 1859-1940. (LČL 3, 2000 : 811) S

Aussi sous le pseudonyme František Pelikán (traductions de J. Verne).

Traducteur de l'anglais et du français (surtout de la littérature d'aventure pour la jeunesse) et écrivain tchèque. Parmi les romans de Verne publiés avant 1900, il a traduit :

Les Tribulations d'un Chinois en Chine, 1879 / O Život, 1900, Vilímek, illustré par L. Benett, autor. překlad

Michel Strogoff, 1876 / Michal Strogov: z Moskvy do Irkutska, 1880-1911, Vilímek, illustré par J. Férat

Le Pays des Fourrures, 1873 / Země kožešin, 1900, Vilímek, illustré par J. Férat et A. de Beaurepaire.

Bohumil Čermák, 1860-1926. $S$

Dans le Dictionnaire encyclopédique de la littérature tchèque (LČL 1, 1985 : 439), on trouve Bohuslav Čermák, 1846-1899, comme traducteur du roman Deux ans de vacances, et non Bohumil Čermák qui toutefois figure dans le catalogue de la Bibliothèque nationale de Prague. Historien et critique littéraire, poète et prosateur, rédacteur, professeur au lycée privé de Prague, employé de la Bibliothèque universitaire de Prague. Parmi les romans de Verne publiés avant 1900, il a traduit :

Deux ans de vacances, 1888 / Dva roky prázdnin, 1890, Vilímek, illustré par L. Benett.

Fr. S. Procházka (František Serafínský Procházka), 1861-1939. (LČL 3, 2000 : 1097-1101) S

Rédacteur du Petit lecteur / Malý čtenár̆ chez Vilímek, il se consacrait surtout à la littérature pour la jeunesse ; depuis 1901 rédacteur de Česká grafická unie et de la revue Zvon (1913-1939) ; rédacteur semi-professionnel (il était aussi employé comme directeur de la Bibliothèque municipale de Prague-Vinohrady) ; traducteur semi-professionnel du français (Verne), de l'allemand (Gerhart Hauptmann) et de l'anglais (Lewis Caroll). Parmi les romans de Verne publiés avant 1900, il a traduit:

Les Indes noires, 1877 / Černá Indie: román, 1893, J. R. Vilímek

Le Château des Carpathes, 1892 / Tajemný hrad v Karpatech: román, 1893, Vilímek, illustré par L. Bénett.

Jiří Stanislav Guth-Jarkovský, 1861-1943. (LČL 1, 1985 : 833-836) O

$\mathrm{Au}$ service d'une famille princière en tant que professeur, il voyage beaucoup ; puis, entre 1888 et 1918, professeur dans les lycées à Prague, depuis 1918 employé au Bureau du Président de la République. Parmi les romans de Verne publiés avant 1900, il a traduit :

Claudius Bombarnac, 1892 / Klaudius Bombarnak, 1893, Vilímek, illustré par L. Benett.

Alois Cerman, 1864-1929. $S$

Parmi les romans de Verne publiés avant 1900, il a traduit : 
La Jangada, huit cents lieues sur l'Amazone, 1881 / Jangada, osm set mil na řece Amazonce, 2 vol., 1883, A. Lauermann

La Jangada, huit cents lieues sur l'Amazone, 1881 / Tajemství pralesa: osm set mil na řece Amazonce, 1895, Vilímek, illustré par L. Benett.

\section{Bedřich Fricke, 1864-1905. S}

Parmi les romans de Verne publiés avant 1900, il a traduit :

Hector Servadac, 1877 / Na kometě, 1896 ou 1897, Vilímek, illustré par P. Philippoteaux

La Maison à vapeur, 1880 / Zemí šelem, 1894-1895, Vilímek, illustré par L. Benett

Sans dessus dessous, 1889 / Zmatek nad zmatek, 1896 ou 1897, Vilímek, illustré par G. Roux.

Josef Pachmayer, 1864-1928. (LČL 3, 2000 : 736-737) S

Journaliste actif - rédacteur de Národní listy, collaborateur de Moderní žena, Večer, etc. Traducteur en neuf langues dont le français (P. Bourget, O. Feuillet, J. et. E. de Goncourt, Maupassant, J. Richepin, V. Sardou) ainsi que de trois autres langues romanes, avec l'anglais (J. F. Cooper, A. C. Doyle), l'allemand, le suédois, le russe, le polonais. Parmi les romans de Verne publiés avant 1900, il a traduit :

Mathias Sandorf, 1885 / Nový hrabě Monte Kristo, Nový hrabě Monte Christo, 1894, Vilímek, illustré par L. Benett (deux variantes du titre dans le Catalogue collectif)

Nový hrabě Monte Kristo, 1894, [Omaha ou « éditeur inconnu »].

Jaroslav Pšenička, 1865-1954. (LČL 3, 2000 : 1155) S

Employé de la poste, auteur des manuels pour les employés de poste, traducteur principalement du français (La Rochefoucauld, Maupassant, Richepin, Verne) et de l'allemand, ayant collaboré par ses traductions des belles-lettres avec les éditeurs Jos. R. Vilímek, J. Otto, mais surtout avec plusieurs journaux quotidiens (Národní Politika, Venkov) et revues (Rozkvět, Vesna, Literární listy). ${ }^{4}$ Parmi les romans de Verne publiés avant 1900, il a traduit :

César Cascabel, 1890 / Oceánem na kře ledové: César Cascabel, 1891, Vilímek

Robur le Conquérant, 1886 / Vzducholodí kolem světa, 1893, Vilímek.

Hugo Kosterka, 1867-1956. (LČL 2, 1993 : 872-873) S

Employé de la Poste, puis au Ministère des Postes ; traducteur du danois, du norvégien, du suédois, de l'espagnol, du portugais et d'autres langues ; à partir du français, il a uniquement traduit le roman de Verne suivant :

Mirifiques aventures du maitre antifer, 1894 / Dobrodružná závět', 1894, Vilímek, illustré par G. Roux, 1850-1929.

\section{Bohumil Klika, 1868-1942. S}

Parmi les romans de Verne publiés avant 1900, il a traduit :

Le Tour du monde en quatre-vingts jours, 1873 / Cesta kolem světa za 80 dní, autoris. překlad B. Kliky, 1900, J. R. Vilímek

Aventures de trois Russes et de trois Anglais dans l'Afrique australe, 1872 / Dobrodružství tř̌ Rusů a tří Angličanů vjižní Africe, illustré par F. Ferat, autoris. překlad, 1900, Vilímek.

\section{J. Tichý. O}

Parmi les romans de Verne publiés avant 1900, il a traduit:

Le Tourdumondeen quatre-vingtsjours, 1873/Cestakolemsvětavosmdesátidnech,1884, A.Lauermann.

\section{Jaroslav Čermák. O}

Parmi les romans de Verne publiés avant 1900, il a traduit :

4 LA PNP Strahov, fonds Jaroslav Pšenička, correspondance de rédaction adressée à J. Pšenička par Sigismund Bouška (« Nový život ») le 15 janv. 1894, František Dlouhý ( Literární listy », « Vesna ») le 4 déc. 1895, František Sekanina ( Éditions J. R. Vilímek », lettre non datée), Václav Beneš (« Národní Politika ») le 24 fév. 1900, J. L. Turnovský (« Nedělní listy ») le 23 avr. 1900, Karel Čvančara (« Venkov », « Rozkvět ») le 5 juil. 1911 et bien d’autres ; toutes les lettres citées concernent les traductions du français de J. Pšenička. 
L'Étoile du sud, 1884 / Hvězda jihu : v zemi diamantů, 1893, Vilímek, illustré par L. Benett

De la Terre à la Lune, 1865 / Do měsíce.

Josef Jan Svátek, 1870-1948. (LČL 4, 2008 : 434-436) P

Neveu de l'écrivain Josef Svátek, 1835-1897. Écrivain, journaliste (rédacteur du journal Pražské novi$n y$ ) et traducteur professionnel du français (traduisant les prosateurs français actuels dès 1890, aussi que les romans d'A. Dumas père dans les années 1930). Parmi les romans de Verne publiés avant 1900, il a traduit :

Nord contre Sud, 1887 / Sever proti Jihu, 1894, Vilímek.

\section{R. Růžička. O}

Parmi les romans de Verne publiés avant 1900, il a traduit :

Nord contre Sud, 1887 / Sever proti Jihu : nejnovějši román od Jules-a Verne-a, 1890, August Geringer, Chicago.

\section{Gustav Janda. $O$}

Parmi les romans de Verne publiés avant 1900, il a traduit :

Un Capitaine de quinze ans, 1878 / Patnáctiletý kapitán, 2 vol., 1884, A. Lauermann.

\section{H.}

Parmi les romans de Verne publiés avant 1900, il a traduit :

Un Hivernage dans les Glaces, 1855, publié dans Le Docteur Ox, 1874 / Zimní pobyt v ledovém moři, 3 éditions, 1876 ou 1877,1886 et 1895 , J. Otto.

J. H.

Parmi les romans de Verne publiés avant 1900, il a traduit :

Le Pays des fourrures, 1873 / Země kožešin, 2 vol., 1876, Spolek pro vydávání laciných knih českých.

\section{L.H.}

Parmi les romans de Verne publiés avant 1900, il a traduit :

Un drame dans les Airs, 1851 / publié dans Le Docteur Ox, 1874 / Drama voblacích, 1884, A. Lauermann.

H.F.

Parmi les romans de Verne publiés avant 1900, il a traduit :

Hector Servadac, 1877 / Světem slunečním, 2 vol., 1882 et 1882-3, A. Lauermann.

\section{J. K. J.}

Parmi les romans de Verne publiés avant 1900, il a traduit :

Voyage au centre de la Terre, 1864 / Cesta do středu Země, 1882, A. Lauermann.

\section{J. T-ý.}

Parmi les romans de Verne publiés avant 1900, il a traduit :

Les tribulations d'un Chinois en Chine, 1879 / Číñanovy trampoty v Číně, 1888, Karel Rathouský, Rychnov n. K.

Vingt mille lieues sous les mers, 1870 / Dvacet tisíc mil pod mořem, 2 vol., 1884, A. Lauermann.

\section{P. J. Š. (voir ci-dessus Pavel J. Šulc)}

Parmi les romans de Verne publiés avant 1900, il a traduit :

Les Aventures du capitaine Hatteras, 1866 / Př́hody kapitána Hatterasa, 3 vol., 1884, A. Lauermann.

\section{R. S.}

Parmi les romans de Verne publiés avant 1900, il a traduit :

Les Enfants du capitaine Grant, 1868 / Děti kapitána Granta, 3 vol., 1883, A. Lauermann.

Traducteurs / Traductions anonymes (dans l'ordre chronologique selon la parution) :

Dans le Catalogue collectif (SKC), on trouve 11 titres ou 16 volumes sans indication sur le traducteur, ne serait-ce que sous forme d'initiales du nom. Dans le cas de certaines traductions parues chez A. Hynek notamment, la date de parution n'est pas indiquée par l'éditeur, mais il est possible de la rétablir grâce au Catalogue collectif. 
Vingt mille lieues sous les mers, 1870 / Dvacet tisíc mil pod mořem, 2 vol., 1877, Nákladem spolku pro vydávání laciných knih českých

Divy světů známých i neznámých : výbor románů, 1883, A. Lauermann

Mathias Sandorf, 1885 / Hrabě Sandorf, 1888, Tiskem a nákladem Národních listů

Vingt mille lieues sous les mers, 1870 / Dvacet tisíc mil pod mořem, Díl I, Díl II, 2 vol., 1893, A. Hynek

Cinq semaines en ballon, 1863 / Pět neděl v balóně: výzkumná cesta tři Angličanů po Africe, 1893,

A. Hynek

Michel Strogoff, 1876 / Carův kurýr, 1894, A. Hynek

Le Château des Carpathes, 1892 / Tajemný hrad v Karpatech : román, 1894, Omaha : Pokrok Západu

Le Chancellor, 1875 / Chancellor : deník cestujícího J. R. Kazallona, autour de 1900, A. Hynek

Les Enfants du capitaine Grant, 1868 / Děti kapitána Granta. Díl 2, Austrálie, Díl 1, Jižní

Amerika, Díl 3. Tỉchý oceán, 3 vol., 1900, A. Hynek

Le Pays des fourrures, 1873 / V zemi kožešin, 2 vol., 1900, A. Hynek

Les Révoltés de la Bounty, 1879 / Buřiči z lodě Bounty : povídka historická, 1900, A. Hynek.

Adaptateurs (7 titres dans l'ordre chronologique selon la parution) :

Antonín Pulda, 1848-1894. Auteur dramatique, acteur, régisseur du Théâtre provisoire et du Théâtre national, pour lesquels il traduisait et adaptait les pièces allemandes et françaises.

Le Tour du monde en quatre-vingts jours, 1874 / Cesta kolem světa za osmdesát dní: velká

výpravná hra s predehrou v pěti jednáních a patnácti obrazích, 1876, Mikuláš a Knap.

P. Š. T-ý. Les Indes noires, 1877 / Černá Indie, 1877, F. Kytka, « dle J. Vernea volně vzdělal P. Š. T-ý ».

P. Š. T-ý. Michel Strogoff, 1876 / Carưv kurýr aneb z Moskvy do Irkutska, 1877, F. Kytka, « dle J. Verne-a vzdělal P. Š. T-ý ».

P. Š. T-ý. Cestování pod mořem, 1877, F. Kytka, «dle J. Verne-a vzdělal P. Š. T-ýy.

Pavel J. Šulc. Un capitaine de quinze ans, 1878 / Patnáctiletý kapitán, « dle J. Vernea pro mládež i dospělé vzdělal », 1879, F. Kytka.

J. Marek. Il a adapté Voyage au centre de la Terre, 1864 / Cesta do středu Země, 1881, F. Kytka.

Ferenc Csepreghy. Michel Strogoff, 1880 / Carův kurýr : velká výpravná hra ve dvanácti obrazech, 1892, l'auteur est à la fois l'éditeur.

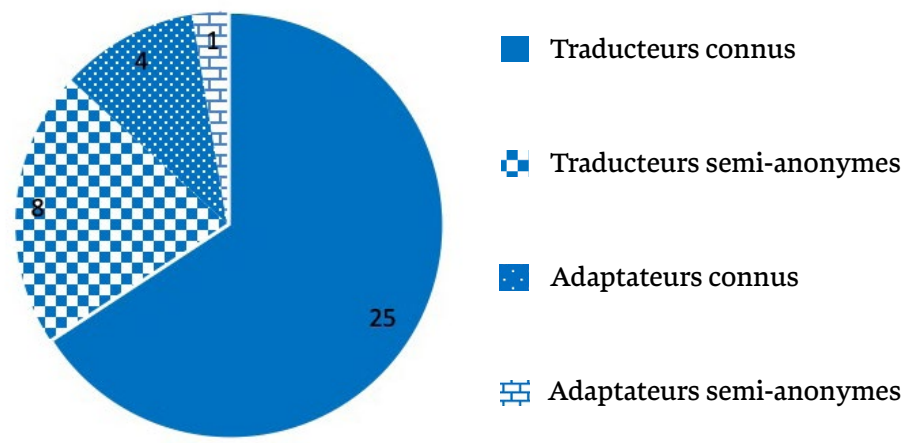

Graphique no 1 : Structure des traducteurs et adaptateurs selon le critère a)

Source : SKC, www.nkp.cz

Les traducteurs (et adaptateurs) de Jules Verne répertoriés entre 1870 et 1900 dont on connaît du moins en partie l'identité sont au nombre de trente-cinq, dont sept restent dans un semi-anonymat (on connaît leurs initiales seulement). Pavel J. Šulc appartient à la fois à la deuxième, la troisième et la quatrième catégorie, puisqu'il est auteur d'une traduction signée par les initiales 
et de quatre adaptations, dont une signée par le nom en entier et trois par des initiales. Comme son identité a pu être rétablie grâce au Dictionnaire encyclopédique (LČL 4, 2008 : 808-809), on le considère également comme un traducteur connu selon le critère a). Le groupe de traducteurs anonymes est constitué probablement par cinq à onze personnes (étant donné que la moyenne par traducteur est d'un ou de deux titres pour le reste du corpus et s'agissant de onze titres anonymes), bien qu'il soit possible qu'il n'y ait qu'un seul traducteur anonyme. Il n'est pas exclu non plus que certains parmi les traducteurs ou adaptateurs connus ou semi-anonymes soient en même temps auteurs d'une ou de plusieurs traductions anonymes. La traduction de la totalité du corpus a donc demandé la participation de trente-cinq à quarante-six personnes.

On peut en déduire que le travail du traducteur n'était pas valorisé systématiquement par tous les éditeurs pragois de l'époque comme une activité créative, exigeant un investissement du potentiel intellectuel. Le fait de publier les traductions anonymes était encore fréquent aux environs de 1900 chez certains éditeurs, plus concrètement dans le cas d'Alois Hynek. Par contre, son principal concurrent dans l'édition d'œuvres de Jules Verne, Josef R. Vilímek, respectait le droit moral de ses traducteurs en imprimant systématiquement leur nom sur la page du titre, où figurait également le nom de l'éditeur et de l'imprimeur. Vilímek veillait aussi au respect des droits des auteurs et des éditeurs d'originaux français, en éditant les « traductions autorisées » (Janeček, 1937 : 64, Horák, 2005 : 30-31).

Dans notre corpus, onze titres différents correspondant à seize volumes traduits sur les quatre-vingt-dix ont des traducteurs anonymes dans le sens où le nom du traducteur n'est pas indiqué, auxquels on peut ajouter dix-huit volumes dans lesquels, il ne l'est que par des initiales. Les volumes traduits sans indication du nom complet du traducteur constituent ainsi 41 \% (34 sur 83) des traductions, et ceux ayant un traducteur connu 59 \% (49 sur 83). Si l'on additionne aux volumes traduits les sept adaptations, qui sont l'œuvre de quatre traducteurs-adaptateurs, et représentent moins de $8 \%$ ( 7 sur 90) du corpus entier, on aboutit aux données indiquées dans le graphique $\mathbf{n}^{\circ} \mathbf{2}$.

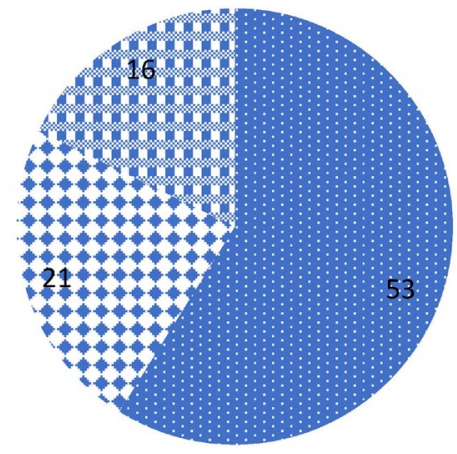

\author{
$\therefore$ Volumes avec traducteur ou \\ adaptateur connu, dont 4 \\ volumes adaptés \\ 4 Volumes avec traducteur ou \\ adaptateur semi-anonyme, dont \\ 3 volumes adaptés \\ Volumes avec traducteur ano- \\ nyme, dont 0 volumes adaptés
}

Graphique no 2 : Nombre de volumes selon la catégorie a) du traducteur Source : SKC, www.nkp.cz

Quant au deuxième critère ou la catégorisation des traducteurs selon la place de la traduction parmi leurs activités professionnelles (qui ne s'applique qu'aux traducteurs connus selon le premier critère), deux traducteurs sont professionnels ( $\mathrm{P}$ dans le tableau ci-dessus), quatorze semi-professionnels (S) et neuf occasionnels (O). Les traductions de Jules Verne étaient 
majoritairement l'œuvre de traducteurs semi-professionnels. Les professions exercées simultanément étaient le plus souvent : rédacteur, journaliste, professeur dans le secondaire, précepteur privé, ou fonctionnaire d'État.

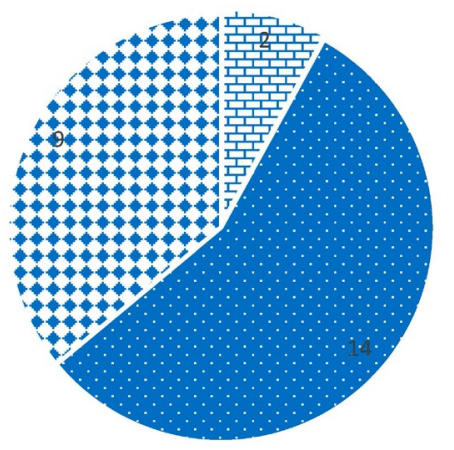

宁 Traducteurs professionnels

$\therefore$ Traducteurs semi-professionnels

.1. Traducteurs occasionnels

Graphique no 3 : Structure des traducteurs selon le critère b)

Source : SKC, www.nkp.cz

Quant au troisième critère ou la catégorisation des traducteurs selon leur place dans le canon littéraire actuel, sur les vingt-cinq traducteurs ${ }^{5}$ connus selon le premier critère, seulement dixsept ont eu droit à une entrée dans le Dictionnaire encyclopédique de la littérature tchèque (LČL 1-4), et on peut donc les considérer comme des traducteurs reconnus, canoniques voire célèbres, puisqu'on a conservé leur mémoire au-delà de plus d'un siècle. Par contre, huit traducteurs sur les vingt-cinq n'ont pas d'entrée dans le Dictionnaire encyclopédique (LČL 1-4) et ne font pas partie du canon littéraire. Ces traducteurs ne se sont pas fait de réputation en tant qu'auteurs ou journalistes : Jan Vincenc Diviš, Alois Cerman, Bedřich Fricke, R. Růžička, Bedřich Janda, Bohumil Klika, J. Tichý, Jaroslav Čermák. Ils sont connus tout au plus par les spécialistes ou les amateurs de l'œuvre de Jules Verne.

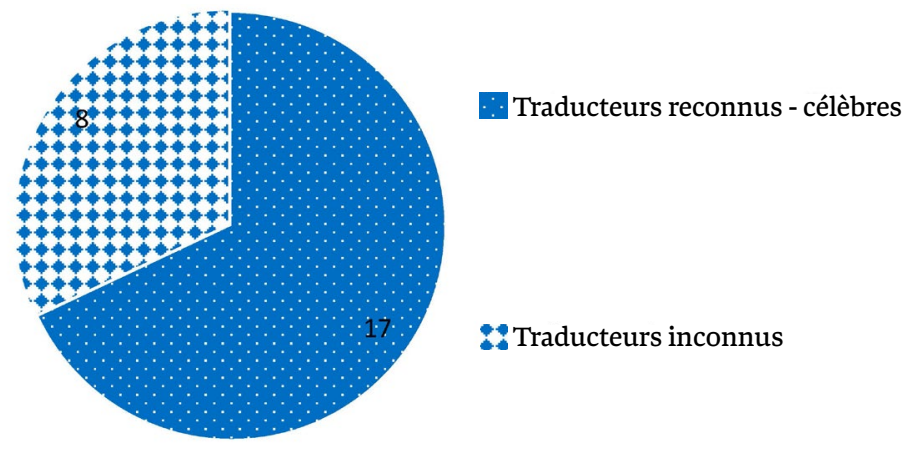

Graphique no 4 : Structure des traducteurs selon le critère c)

Source : SKC, www.nkp.cz

En moyenne pour notre corpus, chaque traducteur se voyait attribué un ou deux titres différents. La plupart des traducteurs - quatorze connus et sept semi-anonymes -, n'ont traduit

Pavel J. Šulc est la même personne que P. Š. T-ý. 
qu'un seul titre, six traducteurs, dont cinq connus et un semi-anonyme en ont traduit deux. Trois traducteurs ont chacun traduit trois titres et deux en ont traduit cinq. Seulement trois traducteurs, Bedřich Fricke (1864-1905), Václav Patejdl (1859-1940) et Jan Wagner (1856-1905), ayant tous travaillé pour Jos. R. Vilímek, ont traduit au moins trois titres différents de Jules Verne sur la période donnée. Pavel J. Šulc (1828-1897) s'est chargé de cinq titres pour le compte de deux éditeurs, quatre pour F. Kytka et un pour Alois R. Lauermann. On ne peut pas observer une véritable spécialisation des traducteurs aux œuvres de Jules Verne, mise à part chez l'éditeur des adaptations F. Kytka et principalement chez l'éditeur des traductions Jos. R. Vilímek, qui avait une position exceptionnelle dans l'introduction de Jules Verne en tchèque dès la fin du $\mathrm{XIX}^{\mathrm{e}}$ siècle.

\section{Conclusion}

Les traductions tchèques de Jules Verne publiées au cours du XIX siècle étaient majoritairement l'œuvre de traducteurs connus (au sens où le traducteur n'est pas resté dans l'anonymat), semi-professionnels et célèbres du point de vue du canon littéraire actuel, au vu des trois critères de catégorisation établis au départ.

Quant au statut du traducteur, il est important d'observer et de constater le taux des traductions anonymes dans le corpus. La visibilité du traducteur et son statut étaient cependant variables suivant les éditeurs. Chez Alois Hynek, les traducteurs des romans examinés sont tous anonymes, par contre chez Josef R. Vilímek, les traducteurs sont tous connus et, à la différence de tous les autres éditeurs, leur nom est de surcroît imprimé sur la page du titre. Vilímek était, par ailleurs, le seul éditeur de notre corpus chez lequel on trouvait le titre original sur la page du titre, imprimé cependant avec une police plus petite que le titre tchèque. Chez d'autres éditeurs, le nom du traducteur est, dans la plupart des cas, indiqué, avec quelques variations (indication du nom par les initiales). Aucune adaptation du corpus n'est complètement anonyme : le nom de l'auteur tchèque de l'adaptation est toujours renseigné, ne serait-ce que par les initiales. La provenance étrangère de tous les titres, traduits ou adaptés, est toujours indiquée, de même que le nom de l'auteur de l'œuvre originale.

La stabilité d'une collaboration entre traducteur et éditeur peut être observée, parmi les traductions de notre corpus, uniquement chez Josef R. Vilímek. 


\section{Bibliographie}

$\checkmark$ Autor:Jules Verne [Auteur:Jules Verne]. https://cs.wikisource.org/wiki/Autor:Jules_Verne [22-02-2018].

$\searrow$ EVEN-ZOHAR, Itamar (1990). « Polysystem Studies ». Poetics Today, International Journal for Theory and Analysis of Literature and Communication, 11.1, pp. 27-51, 73-94, 247-251. https://m.tau.ac.il/ itamarez/ works/books/Even-Zohar_1990--Polysystem\%20studies.pdf [15-01-2018].

У FORST, Vladimír, et al. (1985). Lexikon české literatury: osobnosti, díla instituce $1 A$-G (LČL 1). Praha : Academia.

У FORST, Vladimír, et al. (1993). Lexikon české literatury: osobnosti, díla instituce $2 \mathrm{H}-L$ (LČL 2). Praha : Academia.

У HORÁK, Vadim (2005). Jules Verne v nakladatelství Jos. R. Vilímek. Praha : Thyrsus.

У JANEČEK, Jaroslav (1937). « Jos. R. Vilímek ml., zvelebitel a dovršitel ». In : Karel Sezima (éd.). Jos. R. Vilimek. Osobnost i závod. Praha: Svaz knihkupců a nakladatelů Čs. republiky, pp. 23-82.

У LA PNP [Archives littéraires du Patrimoine littéraire tchèque] Strahov (Prague), fonds Jar. Pšenička, correspondance reçue de S. Bouška le 15 janv. 1894, Fr. Dlouhý le 4 déc. 1895, Fr. Sekanina, lettre non datée, V. Beneš le 24 fév. 1900, J. L. Turnovský le 23 avr. 1900, K. Čvančara le 5 juil. 1911.

У MERHAUT, Luboš, et al. (2008). Lexikon české literatury: osobnosti, díla instituce 4 S-Ž (LČL 4). Praha : Academia.

У OPELÍK, Jiří, et al. (2000). Lexikon české literatury: osobnosti, díla instituce $3 M-\check{R}$ (LČL 3). Praha : Academia.

$\checkmark \quad$ PYM, Anthony (1998). Method in Translation History. Manchester : St. Jerome Publishing.

У RAKovÁ, Zuzana (2011). Francophonie de la population tchèque (1848-2008). Brno : Masarykova univerzita.

У RAKovÁ, Zuzana (2019). « Les premières traductions tchèques de Jules Verne (1870-1900) : archéologie de la traduction ». Studia Romanistica, 19.2, pp. 41-49.

ע Soubornýkatalog ČR [Catalogue collectif tchèque]. www.nkp.cz [27-01-2018].

$\checkmark \quad$ TOURY, Gideon (1978). «The Nature and Role of Norms in Literary Translation ». In : James S. Holmes ; José Lambert ; Raymond van den Broeck : Literature and Translation : New Perspectives in Literary Studies. Leuven : Acco (Academic Publishing Company), pp. 83-100.

$\searrow$ TOURY, Gideon (1995). Descriptive translation studies and beyond. Amsterdam / Philadelphia : John Benjamins Publishing Company. https://doi.org/10.1075/btl.4

У WÖGERBAUER, Michael ; PÍŠA Petr ; šÁMAL Petr ; JANÁČEK Pavel, et al. (2015). V obecném zájmu: cenzura a sociální regulace literatury v moderní české kultuře 1749-2014. Tome I, 1749-1938. Praha : Academia, Ústav pro českou literaturu AV ČR.

\section{Zuzana Raková}

Ústav románských jazyků a literatur

Filozofická fakulta

Masarykova univerzita

Arna Nováka 1

60200 BRNO

République tchèque 